\title{
Improved Neural-aided Sliding Mode Controller for Autolanding under Actuator Failures and Severe Winds
}

\author{
Shaik Ismail ${ }^{1}$ and Abhay A. Pashilkar ${ }^{2}$ \\ National Aerospace Laboratories, Bangalore-560017, India \\ Ramakalyan Ayyagari ${ }^{3}$ \\ National Institute of Technology, Tiruchirappalli-620015, India \\ and \\ Narasimhan Sundararajan ${ }^{4}$ \\ Nanyang Technological University, Singapore 639798
}

\begin{abstract}
In this paper, the fault tolerant capabilities of the neural aided sliding mode controller for autolanding under actuator failures and severe winds developed earlier are improved significantly by incorporating a novel anti-windup strategy and a phase compensation scheme. This controller further increases the size of the fault tolerance envelope for various types of control surface stuck faults and provides complete coverage at every point within the envelope boundaries. Earlier work by the authors showed the existence of a neural-aided sliding mode controller which could handle a wide range of actuator stuck faults. One of the major drawbacks of this earlier controller is that it does not ensure that all points within the range of minimum and maximum bounds of the fault tolerance envelope are covered. The anti-windup proposed in this paper is a generalization of the scheme used for proportionalderivative-integral controllers to the cascaded trajectory following controllers designed by the authors. This scheme can handle requirements of state limiting as well as multiple redundant control surface saturation. The proposed anti-wind up design is a simplification over the command filter approach used for adaptive backstepping. The approach is demonstrated for a fixed-wing aircraft undergoing unknown actuator stuck failures and subject to severe wind disturbances during autolanding. An example of three control surface failures (both ailerons and rudder) handled by this controller is also presented.
\end{abstract}

\section{Introduction}

AULT tolerant control has generated a great deal of interest due to its potential applications for both manned $F$ and unmanned aircraft. Landing is the most critical flight phase for any air vehicle, and in the case of commercial aircraft most of the accidents have occurred during the approach and landing phases although their flight time comprises only a small portion of the total flight phase. Further, almost half of these accidents were due to severe wind turbulences during landing, and the other half due to stuck actuator failures. The probability of the actuator fault during the landing phase is higher compared to that during the cruise phase because the control surfaces of the aircraft are commanded more rapidly and frequently during the landing phase. The failure of the control surfaces during the landing phase causes more fatal accidents than during the cruise phase because there is limited time and space for recovery due to the proximity of the aircraft to the ground. Recourse to autolanding ensures cost reduction, repeatability and safety. The autolanding controller should be robust enough for various disturbances such as wind turbulence, unpredictable gust near the ground, and control surface failures.

At present, a majority of the autolanding controllers are classical PID controllers which perform well under normal landing conditions but, fail under external disturbances like wind shear and turbulence, and aircraft component and actuator failure conditions. Fault Tolerant Control Systems (FTCS) for autolanding aircraft are being

\footnotetext{
${ }^{1}$ Senior Principal Scientist (retd.), Flight Mechanics and Control Division, Post Box No. 1779.

${ }^{2}$ Group Head, Simulation Group, Flight Mechanics and Control Division, Post Box No. 1779.

${ }^{3}$ Professor, Dept. of Instrumentation and Control Engineering.

${ }^{4}$ Professor (retd.), School of Electrical and Electronic Engineering.
} 
extensively investigated [1-2]. Most of the fault tolerant control systems use a Fault Detection and Diagnosis (FDD) module. Using FDD, anticipated faults can be tackled by design. However when a fault which does not belong to the group of faults designed for occurs, a situation may arise where that particular fault is either not detected or correctly identified. In addition to this limitation, FDD schemes need a finite time to become effective, but in that finite time the aircraft stability and performance must be guaranteed. Difficulties also arise when some aircraft parameters need to be identified during flight in real time in the presence of actuator failures or damage to the aircraft structure. In view of all these problems posed by the FDD schemes, a self-learning or adaptive FTCS without FDD is a desirable solution for the autolanding tasks.

At the present time there are three viable solutions that do not explicitly depend on FDD, viz., neural-adaptive control [2], Sliding Mode Control (SMC) [3], and the recently developed L1 Adaptive Control [4]. Neural networks with their ability to approximate nonlinear functions and capability of on-line learning provide a fast mechanism for adapting the aircraft control systems to unknown actuator failures, structural damage and wind disturbances. The ability of SMC to maintain the desired performance of the aircraft in case of unknown actuator faults without requiring an explicit FDD scheme makes it another plausible method for passive fault-tolerant control.

In the early use of neural networks in autolanding controllers, a feed-forward neural network with back propagation learning algorithm was trained off-line to generate the desired trajectories for landing under wind disturbances [5-9]. The advent of Radial Basis Function Neural Network (RBFNN) and a sequential growing and pruning learning algorithm for RBFNN, called as Minimal Resource Allocating Network (MRAN), accelerated the application of neural networks for control of aircraft in general and, autolanding controllers in particular [10-11]. The authors have also addressed the autolanding problem using a completely online neural network controller based on RBFNN in [12].

Extended MRAN (EMRAN) [13] is a faster implementation of MRAN where the parameters of the most dominant neuron only are updated. Based on EMRAN several neural aided controllers were designed for autolanding a typical modern high performance aircraft under severe winds and unknown actuator failures [2, 12, 14-17]. The conventional PID, LQR, $H_{\infty}$ or $H_{2}$ controllers were used as baseline controllers for on-line learning of the networks. It was observed that although the tracking errors of neural aided $H_{\infty}$ controller are much smaller than the neural aided PID controller, the $H_{\infty}$ controller is a more complex higher order controller needing more neurons for the aiding neural network controller [15]. Also, there is no strict theory to guarantee the stability and convergence for the neuro- $H_{\infty}$ control strategy. Thus, the simpler and compact EMRAN aided classical PID controller is a better choice for practical autolanding problems. The control strategy and stability properties of the feedback-error-learning neural controllers are well documented in the literature [16]. Adaptive fuzzy logic based controllers $[14,18]$ have also been successfully implemented for the autolanding problem. An advantage of the fuzzy logic based approach is that the number of parameters required to undertake the controller design are relatively few.

A glaring deficiency of EMRAN aided conventional PID controllers [2] is the presence of "holes" or discontinuities in the fault tolerance envelopes of the controllers. Initial efforts to fill in the gaps in the fault tolerance regions of the neural-aided controllers revealed that the rate and position saturation of the healthy actuators resulted in loss of control and failure to complete the autolanding task within the tight specifications and, hence discontinuities occur in the fault tolerance range of the controller. Training signal hedging (TSH) [19] and pseudo control hedging (PCH) [20] are two well known methods for handling actuator position and rate limits. The command filter approach [21] is yet another technique which was developed to address this issue by introducing a second order filter with rate and position limits between each of the cascaded stages in a backstepping controller. An advantage of the command filter approach is the ability to handle the limiting of control surface signals as well as states. The additional benefit is the ability to generate and use the derivative of the command from the outer loop and use it in the inner loop inversion.

The objective of the present work is not only to expand the fault tolerance envelope of the controller without gaps, but also to enable the controller to handle more types of actuator failures. As a first step in achieving this objective, a basic controller based on the principles of time-scale separation and Nonlinear Dynamic Inversion (NDI) is designed [22]. This fault tolerance baseline controller was augmented with a combination of an EMRAN neural controller and an SMC controller [23-24]. The basic motivation behind introducing both EMRAN and SMC controllers is as follows:

a) Any control design technique which uses either a FDD approach will have to consider the finite time it takes to detect the failure and reconfigure the controller. Similarly, controllers that use online learning strategy, must also factor in the finite time it takes the adaptive controller to learn the changed dynamics. During this time, the controller must be able to retain stability under the failure condition. 
b) The advantage of a sliding mode controller is that it can act immediately based only on the direction of the error, thereby arresting any instability. However, the disadvantage of SMC is that it results in large high frequency commands to the actuator and can cause chattering.

c) A neural network which learns the inverse dynamics at a moderate pace is desirable in order to recover from the failure and achieve improved post-failure accuracy in terms of landing on the touchdown point. The neural controller is also capable of generating high frequency command signals as it is based on direct dynamic inversion.

The authors previous work [24], first proposed the basic approach outlined above and a theoretical justification is given in this paper based on Lyapunov arguments. The resulting controller schematic is shown in Fig. 1. It is seen in this figure that the baseline classical feedback controller (FC) is supported by the sliding mode controller (SMC) and subsequently by the neural network controller (EMRAN). The FC and SMC produce commands at the level of each of the rotational axes (pitch, roll and yaw). These commands are then allocated to the multiple control surfaces present on this aircraft via a static control allocation matrix. The EMRAN controller on the other hand has the ability to learn the inverse dynamics and therefore it is designed to independently compute all the control surface deflections.

Since the controller is autonomous, a Tracking Command Generator (TCG) is required. The TCG determines the offset of the aircraft from the desired ground track for each segment of flight which can be approximated by straight lines or arcs of circles, and generates command signals which are input to the Feedback Controller (FC). The reference commands consist of altitude $\left(h_{r e f}\right)$, velocity $\left(V_{r e f}\right)$, cross distance from the desired track vector $\left(\delta_{\text {ref }}\right)$ and the angular error of the aircraft velocity vector from the desired track vector $\left(\psi_{\text {ref }}\right)$. It is assumed that the landing trajectory can be divided into straight line and turn segments. The generation of the tracking commands is briefly described in [2].

The first problem we noticed in using this controller was that for some failure cases, its performance was worse than that of the baseline controller. Secondly, one expects that a well designed controller will be able to handle any actuator stuck positions which lie between a certain minimum and maximum value. However, in practice it was found that the failure tolerance envelope exhibited gaps for this controller [23] within the outer bounds of its fault tolerance envelope. Detailed examination of these specific cases showed that the controller was operating very close to either the position or rate limits of one or more control surface actuators resulting in a failure to meet the strict touchdown criteria. Therefore, it was mandatory to address the issue of rate and position limits of the actuator in the presence of the neural and sliding mode components in the controller to remove the gaps.

It may be noted that our aircraft model is unstable in longitudinal axis. Therefore, position saturation of the elevators for a few seconds causes open loop divergence of the plant. This means that only short duration of position or rate saturation of the elevators can be tolerated. This aspect combined with the moderate learning rate that we use in our neural network as described above, does not require us to make any special provisions within the neural controller with regard to learnability. In other words, the neural controller is always learning the inverse dynamics at a moderate rate, irrespective of whether the actuator is in saturation or not.

Our baseline NDI controller is designed with time scale separation. Thus, the second order command filters proposed in [21] between each stage of the cascaded controller was unnecessary for our controller. Therefore, phase compensating filters are sufficient to mitigate the phase lag caused by actuator rate saturation, and anti-windup strategies are used to prevent over-driving the actuators when they are in position saturation. The anti-windup and phase compensation block are shown in Fig. 1. This results in a simpler autolanding controller that can accommodate adverse effects of unknown actuator failures and severe wind disturbances for fixed wing aircraft.

The performance of the autolanding controller was evaluated using the six degrees of freedom (6 DOF) simulation of a benchmark autolanding problem formulated by [2]. The mathematical model of a typical modern high performance fighter aircraft used for simulation has independent elevator and aileron control surfaces. It is shown that the autolanding controller designed in the present study can handle more (six-) types of actuator failures: failure of left-elevator, failure of left-aileron, simultaneous failure of left-elevator and left-aileron, simultaneous failure of left-elevator and right-aileron, simultaneous failure of both the ailerons, and failure of rudder. Further, it is shown that the autolanding controller has wider fault-tolerance range for all the six-types of actuator failure cases studied. Thus, the autolanding controller discussed in the present paper is the most robust controller designed so far for the benchmark autolanding problem chosen for study by the authors.

The rest of the paper is organized as follows: Section II describes the formulation of the autolanding problem, including the mathematical models of the aircraft and actuators, types of actuator failures, the landing trajectory, wind profiles during the landing task, the performance and safety criteria, and the definition of fault-tolerance feasibility ranges for various types of actuator failures. The design of the neural-aided hybrid autolanding controller 
is discussed in Section III. For the sake of simplicity, this controller will be simply called as Neuro-Sliding-Mode Backstepping Controller (NSBC) in the rest of the paper. The performance of the improved autolanding controller for various types of actuator failures is discussed in Section IV. Finally, Section V discusses the conclusions drawn from the present work, and the directions for future research.

\section{Autolanding Problem Formulation}

\subsection{Aircraft Model}

The aircraft model chosen for the present study has flight dynamics similar to a modern high performance fighter aircraft [25], but with independent left and right elevator and aileron controls. Computational Fluid Dynamics (CFD) method was used to generate additional aerodynamic data for the split elevator and aileron control surfaces. The aerodynamic model also incorporates the ground effects and the landing gear effects. The engine model (with a 5sec first order lag representing the dynamics) completes the 6 DOF simulation model. The two elevators can be moved together or in differential mode $(-25$ to +25 deg.). The deflection range for the independent ailerons is -20 to +20 deg., and for the rudder it is -30 to +30 deg.

As the first step, a linear model of the aircraft is required for the linear backstepping controller design. The nonlinear model of the aircraft was trimmed at the straight and level flight condition (vel $=82.66 \mathrm{~m} / \mathrm{s}$, alt $=600 \mathrm{~m}$ ). The linear model at this flight condition has been used in the design of the NDI based flight controller (FC). The design process is described in detail in [22].

\subsection{Actuator Models and Failure Types}

The hydraulic actuators are modeled as first order lags with a time constant of $50 \mathrm{~ms}$, and a rate limit of 60 $\mathrm{deg} / \mathrm{s}$. In this paper the failure envelopes of six types of failures are studied: failure of either left- or right-elevator alone, failure of either left- or right-aileron alone, simultaneous failure of left-elevator and left-aileron, simultaneous failure of left- elevator and right-aileron, simultaneous failure of both the ailerons, and failure of rudder. Failure of both elevators is not considered because this case is, in general, not recoverable. A particular case of three simultaneous failures (two ailerons and rudder) is also presented to demonstrate the robustness of the control scheme.

Failure of actuators can occur at any time during the flight. In the present study failures were injected just before the two critical stages of the landing flight: level turn and descent phases. Further, the hard over positions of the failed control surfaces can take any value within the permissible range of deflections.

\subsection{Landing Trajectory}

The autolanding trajectory chosen for study is same as that found in [23]. The trajectory consists of flight segments such as wings-level flight, coordinated turns, glide slope descent and finally the flare maneuver and touch down on the runway. The landing is simulated under severe winds which cause deviations of the aircraft from the specified trajectory.

\subsection{Wind Profiles}

Severe wind disturbances are assumed to be present along all the three axes throughout the landing mission, and are modeled on the Dryden spectrum [23]. The aircraft model sees these wind components in the earth axis. These are transformed appropriately in the body axes and used in the simulation.

\subsection{Safety and Performance Criteria}

The desired touchdown point of aircraft under normal operating conditions is $x=0 m, y=0 m, z=0 m$. Since this ideal touchdown cannot be achieved under unknown actuator failures, some safety criteria that need to be satisfied during final phase of landing are specified as given below:

- X-distance: $-100 m \leq x \leq 400 m$ and Y-distance: $-5 m \leq y \leq 5 m$, to restrict the landing area to a rectangle of $500 m \times 10 m$, also called as "Pill Box"

- Total velocity: $V_{T} \geq 60 \mathrm{~m} / \mathrm{s}$ to prevent stall

- Sink rate: $h \geq-2.0 m / s$, to prevent landing gear damage

- Bank angle: $|\phi| \leq 10 \mathrm{deg}$, to prevent wing tips touching the ground

- Heading angle error: $|\psi| \leq 15$ deg , to prevent excessive side load on landing gear 


\subsection{Fault-tolerance Envelopes}

It is obvious that all the possible actuator failures cannot be accommodated by any controller because in some cases the resulting moments cannot be trimmed out for the landing maneuver, that is, a steady level turn or wing level descent may not be possible. Thus, the full range of hard over positions must be checked for the feasible subset. This procedure is described in detail on [23].

\section{Improved Autolanding Controller}

The philosophy behind the design of the improved autolanding controller discussed in this paper can be illustrated using the elegant ideas inherent in the Sliding Mode Control (SMC) concept. Let an affine plant be represented by

$$
\dot{x}=f(x)+b u
$$

where, $x \in R$ is the sate vector and $u \in R$ is the control input. Let the sliding mode surface be given by

$$
S=\tilde{x}+\lambda \int_{0}^{t} \tilde{x} d \tau
$$

where, $\tilde{x}=x-x^{d}$ is the state error, with $x^{d}$ being the desired trajectory. The sliding mode control law is composed of two modes. The first mode is a reaching mode where the states beginning from arbitrary state are attracted towards the sliding surface $S=0$. In the second mode, the states slide along the sliding surface. In this mode, we set the time derivative of sliding surface $\dot{S}=0$. It will be shown by means of a Lyapunov proof that the control signal to acquire and move on the sliding surface is given by

$$
\begin{aligned}
u & =-\frac{1}{b} \lambda \tilde{x}+\frac{1}{b}\left\{\dot{x}^{d}-f(x)\right\}+K \operatorname{sat}(S) \\
& =u_{F C}+u_{N N}+u_{S A T}
\end{aligned}
$$

Consider the candidate Lyapunov function $V=S^{2} / 2$ whose time derivative is given by

$$
\dot{V}=\left(f(x)+b u-\dot{x}^{d}+\lambda \tilde{x}\right) \cdot S
$$

The dynamic inversion control will not in general produce an exact estimate of the nonlinear dynamic term $f(x)$. Therefore, the equivalent control term may be written as

$$
u=\frac{1}{b}\left\{\dot{x}^{d}-\lambda \tilde{x}-\hat{f}(x)\right\}+K \operatorname{sat}(S)
$$

Inserting (5) into (4), we have

$$
\begin{aligned}
\dot{V} & =(f(x)-\hat{f}(x)-b \cdot K \operatorname{sat}(S)) \cdot S \\
& =(f(x)-\hat{f}(x)) \cdot S-(b \cdot K|S|)
\end{aligned}
$$

To guarantee system stability, $\dot{V}$ must satisfy the sliding condition inequality $(S \cdot \dot{S}<0)$. Therefore, we can specify $K$ to be such that

$$
b \cdot K=|f(x)-\hat{f}(x)|+\eta
$$


It is noted that the term $(f(x)-\hat{f}(x))$ denotes the deviation of the actual model dynamics from the estimated dynamics. The resulting controller leads to

$$
\dot{V} \leq-\eta|S|
$$

According to the definition of Lyapunov stability, the above result assures that the controlled system is stable. The control law represented by (3) represents a variable structure controller. The first term $\left(u_{F C}\right)$ can be viewed as a classical feedback controller with the feedback gain $-(1 / b) * \lambda$ multiplying the state error. The second term $\left(u_{N N}\right)$ could be a controller based on Nonlinear Dynamic Inversion (NDI) because it is the exact inverse of (1). In our case we will use a neural controller EMRAN with online learning which is based on radial basis functions [13]. Finally, the third term $\left(u_{S A T}\right)$ is the saturation control signal which fires when the error exceeds the error threshold around the sliding surface. The function 'sat' operates on the sliding surface to generate a signal in the range [-1, 1]. Typical function could be a deadband with width of the error threshold or a linear variation with unity slope. The resulting controller schematic is shown in Fig. 1. The outermost loop is the tracking controller which computes the desired ground track angle, lateral deviation from the desired trajectory, desired velocity, and the desired altitude [2]. In a neural-aided controller, the rate of learning of the neural network can be increased for faster recovery from failures. But, high rates of learning may excite unmodelled dynamics leading to stability problems. However, instead of increasing the rate of learning or choosing higher gains within the baseline controller, a sliding mode controller which comes into play when large feedback errors are detected signaling failures is used. The design of the individual blocks of the controller is briefly discussed in the following sections.

\subsection{Feedback Controller (FC)}

The classical feedback controller (FC) is designed for nominal plant, separately for the longitudinal and lateraldirectional axes [22]. The feedback gains are chosen such that when there are no failures or winds the innermost feedback loops do not cause rate or position limiting of the actuators. The design of the FC controller is in fact based on the time-scale separated NDI principles.

The design begins by considering the three rotational axes for feedback control. As described in [22], the dependent variables of the aircraft chosen for control law design are $y=\left[\begin{array}{llll}\alpha & q & \beta & p\end{array}\right]^{T}$, and the inner loop states are $x=\left[\begin{array}{lll}a & p & r\end{array}\right]^{T}$. To simplify the controller design, the roll and yaw rates are transformed from the body axis to the stability axis using the transformation:

$$
\left[\begin{array}{c}
p_{s} \\
r_{s}
\end{array}\right]=\left[\begin{array}{cc}
\cos \alpha & \sin \alpha \\
-\sin \alpha & \cos \alpha
\end{array}\right]\left[\begin{array}{l}
p \\
r
\end{array}\right]
$$

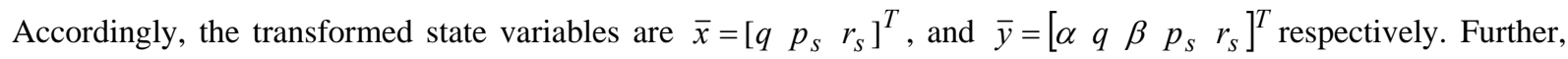
multiple surface redundancy is used to enhance the ability of the controller to handle failures. Using a ganging matrix, the control inputs $u=\left[\begin{array}{lllll}\delta_{e-l e f t} & \delta_{e-r i g h t} & \delta_{a-l e f t} & \delta_{a-r i g h t} & \delta_{r}\end{array}\right]^{T}$ are transformed into three pseudo controls $\bar{u}=\left[\begin{array}{lll}\delta_{\text {pitch }} & \delta_{\text {roll }} & \delta_{\text {yaw }}\end{array}\right]^{T}$ to exercise decoupled control of each of the three rotational axes as shown below:

$$
u=\left[\begin{array}{ccc}
1 & -\mathrm{K}_{\text {aei }} & -\mathrm{K}_{\text {rei }} \\
1 & \mathrm{~K}_{\text {aei }} & \mathrm{K}_{\text {rei }} \\
0 & -1 & 0 \\
0 & 1 & 0 \\
0 & \mathrm{~K}_{\text {ari }} & 1
\end{array}\right] \bar{u}
$$


where, $K_{a r i}$ is the well known aileron to rudder interconnect. The gains $K_{a e i}$ and $K_{r e i}$ are introduced to exploit the capability of the elevators in differential mode to generate additional rolling and yawing moments respectively. The gain $K_{a e i}$ enables the controller to handle a new type of failure (namely failure of both the ailerons), and the gain $K_{\text {rei }}$ allows the controller to handle a larger range of rudder failures. Proper values were chosen for the gains ( $K_{a r i}=1.2 \mathrm{deg} / \mathrm{deg}, K_{a e i}=0.75 \mathrm{deg} / \mathrm{deg}$, and $K_{r e i}=0.27 \mathrm{deg} / \mathrm{deg}$ ) so that control decoupling of pitch, roll and yaw axes is achieved [23]. The control allocation matrix block represented by (10) is shown in Fig. 1.

The principal aim of the autolanding controller is to reject winds and follow the predefined inertial trajectory for autolanding. Therefore, it seems appropriate to replace whenever possible the air-data feedback of angle of attack and sideslip with physically similar inertial signals. In the case of angle of attack, we have considered the pitch attitude for feedback. However, in case of the sideslip, placement of a low pass filter, with a first order time constant of 30ms, in the feedback loop seemed to be the most appropriate solution.

It is to be noted in the feedback controller design that the angle of attack figures in the transformation of the body axis roll and yaw rates to the stability axis roll and yaw rates. If the measured value of the angle of attack is used for this transformation, it creates a coupling between the longitudinal and lateral-directional axes. This is avoided by using the nominal 1-g angle of attack for the body-to-stability axis transformation.

The conventional washout filters (WO), and PID blocks are included in the FC controller to enhance the ramp following of the control system to altitude and track angle. This is achieved by adding a signal proportional to the derivative of the altitude and track angle commands respectively. The derivative is constructed using a washout filter. The airspeed loop also has an additional lead-lag compensator to improve the speed of response without compromising the overshoot. The final longitudinal and lateral-directional axis FC design is presented in Fig. 2 and Fig. 3 respectively.

\subsection{Sliding Mode Controller}

The main task of this controller is to recover from failure using, if required, the full authority of the control surfaces which are healthy. In the present work the utility of a Sliding Mode Controller (SMC) is proposed for this purpose.

The saturation control of the sliding mode controller comes into play only if the error exceeds a threshold. The saturation control is designed to act rapidly once a stuck actuator failure results in an error exceeding this threshold. This is aimed at preventing the aircraft from seeing large transients and permitting EMRAN controller to learn at a moderate rate. The saturating controller is implemented in the longitudinal axis only.

\subsection{EMRAN Controller}

The fault tolerance capability of the feedback controller can be enhanced with the addition of a neural controller. The EMRAN neural network is chosen to aid the FC because of its fast and efficient on-line learning abilities. The FC controller is not only used to stabilize the system but also to provide signals to train the EMRAN network online. The EMRAN controller uses the reference signals from the Tracking Command Generator, and the aircraft outputs to generate its command signals. The sum of the command signals from EMRAN, FC and the Saturation Controller are used to drive the actuators. The EMRAN learns the aircraft inverse by observing the total control signal to the actuators and comparing it to its own output. In the process, it attempts to drive the outputs from the other controllers to zero. The schematic of the combined longitudinal and lateral-directional EMRAN block is shown in Fig. 4. The state variables are scaled by the linear derivatives to improve the numerical conditioning of the inputs to the EMRAN block.

\subsection{State and Control Limiting Scheme}

A control surface saturation detection mechanism is introduced in the feedback controller to protect against integrator windup. From the control allocation matrix given in (5), it can be inferred that the saturation of the left elevator could be due to the pitch, roll, or yaw axis control signals. Therefore, in this case we hold the integrators in all the three axes for the duration for which the left elevator is saturated. In this way, we prevent the control system from overdriving the actuators.

Limits on the state variables like pitch attitude is also incorporated in the pitch and the roll axis integrators. The general principle for the anti-windup design is that any state or control surface saturation in an inner loop of the cascaded controller should result in the integrators in the outer loops to be held for the duration of the time the 
variable is in saturation. This general anti-windup scheme addresses both state and control surface saturation for a cascaded controller structure. Our scheme is simpler that the TSH, PCH and Command Filtering schemes used in literature.

\subsection{Phase Compensators}

The SAAB phase compensator [26] is used to reduce the phase lag due to rate limiting. The schematic of the compensator is shown in Fig. 5. The filter time constants are chosen for the autolanding task, and it is observed that better results are obtained when the phase compensator is placed ahead of the actuator. The frequency response of the compensated actuator is shown in Fig. 6.

The fault tolerance performance of the improved autolanding controller is evaluated by six degree-of-freedom (6 DOF) simulation of the auto landing task under various types of actuator failures and wind disturbances.

\section{Six DOF Simulation of Autolanding Task}

The fault tolerance performance of our earlier autolanding controller based on classical PID control, SMC, and EMRAN augmentation (BTFC+SMC+EMRAN) was discussed in [24]. The fault tolerance capability of our present autolanding controller is much superior to the earlier design. Further, a comparison of the fault tolerance performance of the classical Feedback Controller (FC) alone, with that of EMRAN and SMC augmented controller (FC+SMC+EMRAN) was reported in [23]. It was observed that the failure tolerance performance of FC was being degraded by EMRAN+SMC augmentation, especially in the case of failure of both the ailerons. This anomaly was due the actuator rate and position limiting effects which were affecting on-line inverse dynamics learning capability of the EMRAN controller. This anomaly is removed in the present design using anti-windup and phase compensation filter in the command path for each of the aerodynamic control surfaces. The performance of the improved autolanding controller in the case of simultaneous failure of both the ailerons is discussed in the following sections. It is further shown that the improved autolanding controller can accommodate simultaneous failure of three actuators as long as the failures are small.

\subsection{Comparison with Earlier Controller}

A comparison of the fault tolerance performance of the autolanding controller discussed in this paper (FC+SMC+EMRAN+SAAB+AWU) with that of our earlier design (BTFC+SMC+EMRAN) [24] is shown in Figs. 7-8 for the case of left-aileron failure and simultaneous failure of left-elevator and left-aileron respectively. It is evident from Fig. 7 that the fault tolerance feasibility map of the BTFC+SMC+EMRAN controller while covering substantial part of the feasible region does have gaps at -11 and -17 degrees of left aileron stuck positions. Also the +20deg aileron stuck position is not part of the fault envelope. On the other hand, the failure tolerance envelope occupies the entire feasible region. Also from Fig. 8 we find that the feasibility envelope for the two surface failure case is much larger than those of our earlier controller [24].

\subsection{Failure of Both Ailerons}

The BTFC design presented earlier [24] is not capable of handling simultaneous failure of two ailerons. The fault tolerance envelope for the case of simultaneous failure of both the ailerons for FC+EMRAN+SMC+SAAB+AWU is same as that for the FC shown in [23]. It is seen that the anti-windup and phase compensation schemes allow the EMRAN controller learn the inverse dynamics smoothly.

\subsection{Three Actuator Failure Case}

The case of simultaneous failure of three actuators is also studied. It is assumed that the left elevator, the right aileron, and the rudder are stuck at $2 \mathrm{deg},-2 \mathrm{deg}$, and 4 deg respectively during level turn. The longitudinal and lateral-directional responses are shown in Fig. 9. It is seen that the sideslip is about 3degrees subsequent to failure and remains at this value during touchdown as well. There are multiple solutions for landing in cross-winds, particularly for aircraft such as ours which has a stable dutch-roll mode. At one extreme, we have the zero sideslip solution with non-zero bank angle (i.e., crabbed landing), while at the other end we can also have a non-zero sideslip with zero bank angle. In this particular case, with three simultaneous control surfaces stuck the controller is able to find a solution which is in between these extremes. Similarly, the velocity shows significant variations due to the 
severe vertical winds (representing a micro-burst) particularly just before touchdown. That the controller is able to handle this case attests to its robustness with respect to unknown failures and severe winds. The case of three actuator failure will be studied further systematically.

\section{Conclusions}

In feedback error learning, a baseline controller frequently designed using classical methods, is aided by neural and sliding mode controllers in order to handle faults. In this architecture, the baseline controller (designed using NDI principles) is protected against actuator position saturation as well as excursions into parts of the state space where controllability is lost using a novel anti-windup scheme. The anti-windup takes into account multiple redundant control surface saturation commonly found in flight control. The proposed anti-windup is a generalization of the scheme used for conventional PID controllers.

Neural and sliding mode controllers generate high frequency large control inputs in response to actuator faults. Therefore, these controllers can benefit from the use of phase compensation filters routinely used in flight control to overcome limitation of actuator rates.

The novel anti-windup in conjunction with a phase compensation scheme has been applied to a neural-aided sliding mode controller designed by the authors to study its tolerance for stuck actuator faults during an autolanding scenario. It is shown that the addition of anti-windup and phase compensation significantly enhances the fault tolerance capability of the neural-aided sliding mode controller.

\section{Acknowledgments}

The authors express their sincere thanks to the organizations NAL-CSIR Bangalore, and NIT Trichy for their encouragement throughout the course of this work.

\section{References}

[1] C. Edwards, T. Lombaerts, H. Smaili, (eds.), Fault Tolerant Flight Control: A Benchmark Challenge, $1^{\text {st }}$ ed., Springer-Verlag, New York, 2010.

[2] A. A. Pashilkar, N. Sundararajan, P. Saratchandran, A fault-tolerant neural aided controller for aircraft auto-landing, Aerospace Science \& Technology, 10 (2006), 49-61.

[3] H. Alwi, Fault Tolerant Sliding Mode Control Schemes with Aerospace Applications, Ph.D. Dissertation, University of Leicester, UK, 2008.

[4] V. V. Patel, C. Cao, N. Hovakimyan, K. A. Wise, E. Lavretsky, $\mathrm{L}_{1}$ adaptive controller for tailless unstable aircraft in the presence of unknown actuator failures, International Journal of Control, 82 (2009) 705-720.

[5] C. C. Jorgensen, C. Scheley, Neural network basedline problem for control of aircraft flare and touchdown, in: Neural Networks for Control, $2^{\text {nd }}$ ed., MIT Press, Cambridge, MA, 1990, pp. 402 - 425.

[6] R. M. Sanner, J-J. E. Slotine, Gaussian networks for direct adaptive control, IEEE Trans. on Neural Networks, 3 (1992) 837863.

[7] Y. Iiguni, H. Akiyoshi, N. Adachi, An intelligent landing system based on a human skill model, IEEE Trans. on Aerospace and Electronic Systems, 34 (1998) 877-882.

[8] G. Saini, S. N. Balakrishnan, Adaptive critic based neurocontroller for autolanding of aircrafts with varying glideslopes, Proc. of Intl. Conference on Neural Networks, 4 (1997) 2288 - 2293.

[9] S. Suresh, S. N. Omkar, V. Mani, N. Sundararajan, Nonlinear adaptive neural controller for unstable aircraft, Journal of Guidance, Control and Dynamics, 28 (2005) 1103-1111.

[10] Y. Lu, N. Sundararajan, P. Saratchandran, Performance evaluation of a sequential minimal radial basis function (RBF) network algorithm, IEEE Trans. on Neural Networks, 9 (1998) 308 - 318.

[11] N. Sundararajan, P. Saratchandran, Y. Li, Fully Tuned Radial Basis Function Neural Networks for Flight Control, Kulwer Academic Publishers, Boston, 2001.

[12] A. A. Pashilkar, N. Sundararajan, P. Saratchandran, Adaptive nonlinear neural controller for aircraft under actuator failures, Journal of Guidance, Control and Dynamics, 30 (2007) 835-847.

[13] Y. Li, N. Sundararajan, P. Saratchandran, Analysis of minimal radial basis function network algorithm for real-time identification of nonlinear dynamic systems, IEEE Proc. - Control Theory and Applications, 147 (2000) 476 - 484.

[14] W. Zhang, Control of a high performance aircraft by using $H_{\infty}$ theory and neural-fuzzy concepts, M. Engg. Thesis, School of EEE, NTU, Sigapore, 1997.

[15] Y. Li, N. Sundararajan, P. Saratchandran, Z. Wang, Robust neuro- $H_{\infty}$ controller design for aircraft auto-landing, IEEE Trans. on Aerospace and Electronic Systems, 40 (2004) 158 - 167.

[16] Y. Li, N. Sundararajan, P. Saratchandran, Neuro-controller design for nonlinear fighter aircraft maneuver using fully tuned RBF networks, Automatica, 37 (2001) 1293-1301. 
[17] Z. Wang, G. Xiong, Neuro-aided $H_{2}$ controller design for aircraft under actuator failure, Proc. of the $20112^{\text {nd }}$ Intl. Congress on Computer Applications and Computational Science, Vol. 2, 2011.

[18] H. -J. Rong, N. Sundararajan, P. Saratchandran, Adaptive fuzzy fault-tolerant controller for aircraft autolanding under failures, IEEE Trans. on Aerospace and Electronic Systems, 43 (2007) 1586 - 1602.

[19] A. M. Annaswamy and J. E. Wong, Adaptive control in the presence of saturation nonlinearity, International Journal of Adaptive Control and Signal Processing, 11 (1997) 3-19.

[20] E. Johnson, A. J. Calise, Neural network adaptive control systems with input saturation, Proc. of the American Control Conference, Vol. 5, 2001.

[21] J. Farrell, M. Sharma, M. Polycarpou, On-line approximation based control of uncertain nonlinear systems with magnitude, rate and bandwidth constraints on the states and actuators, Proc. of the American Control Conference, Vol. 3, 2004.

[22] A. A. Pashilkar, S. Ismail, R. Ayyagari, N. Sundararajan, Design of a nonlinear dynamic inversion controller for trajectory following and maneuvering fixed wing aircraft, Proc. of IEEE SSCI-CISDA 2013, Singapore, 16-19 April 2013, pp.64-71.

[23] S. Ismail, A. A. Pashilkar, R. Ayyagari, N. Sundararajan, Improved autolanding controller for aircraft encountering unknown actuator failures, Proc. of IEEE SSCI-CISDA 2013, Singapore, 16-19 April 2013, pp.96-103.

[24] S. Ismail, A. A. Pashilkar, R. Ayyagari, Guaranteed stability and improved performance against actuator failures using neural-aided sliding mode controller for autolanding task, Proc. of IFAC-EGNAC 2012 Workshop, Indian Institute of Science, Bangalore, 13-15 Feb. 2012.

[25] L. T. Nguyen, M. E. Ogburn, W. P. Gilbert, K. S. Kibler, P. W. Brown, P. L. Deal, Simulator study of stall/post-stall characteristics of a fighter airplane with relaxed longitudinal static stability, NASA TP-1538, 1979.

[26] L. Rundqwist, Phase compensation of rate limiters in unstable aircraft, Proc. of the IEEE Intl. Conf. on Control Applications, 15-18 Sep. 1996, pp. 19-24. 


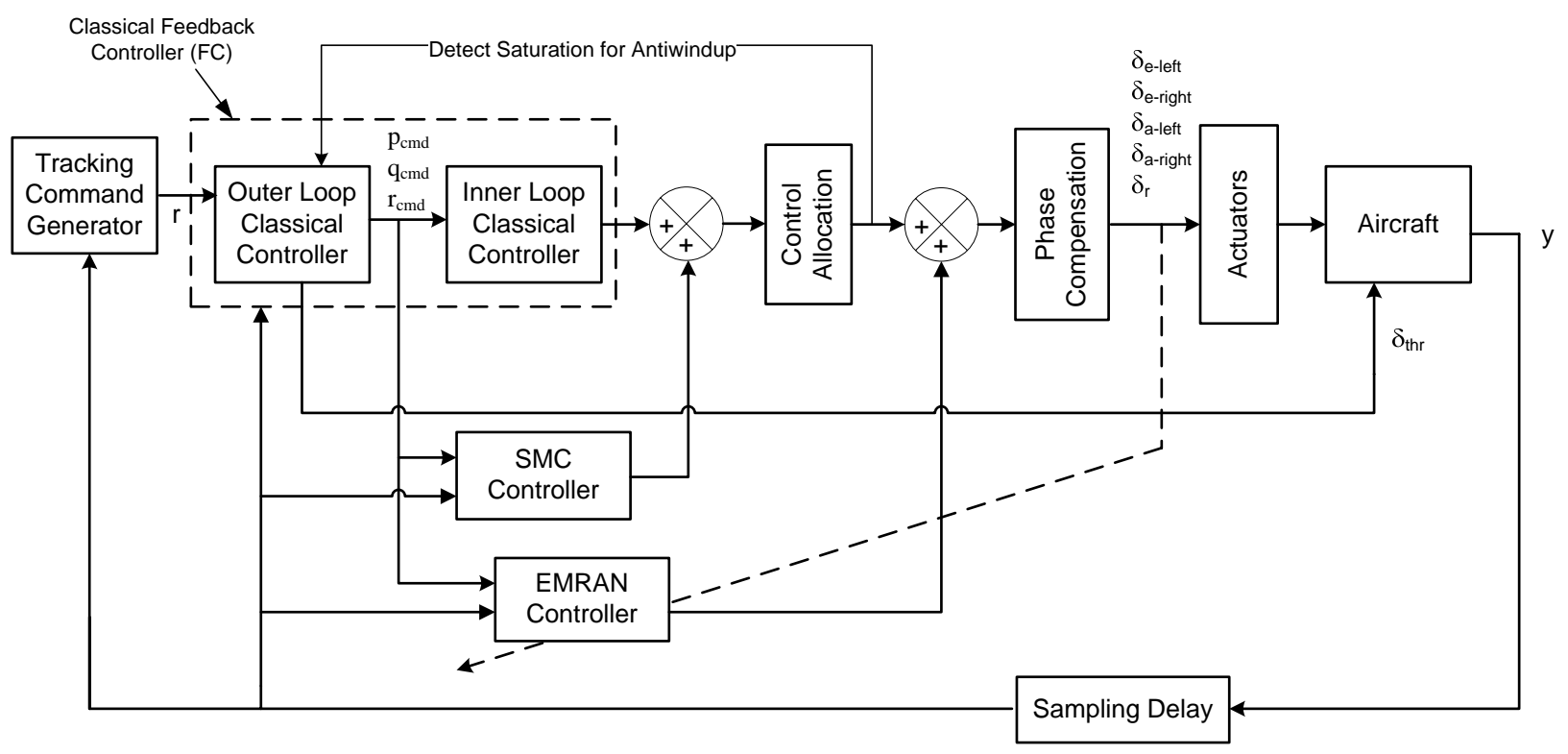

Fig. 1 Schematic of Neural Sliding Mode Flight Controller 


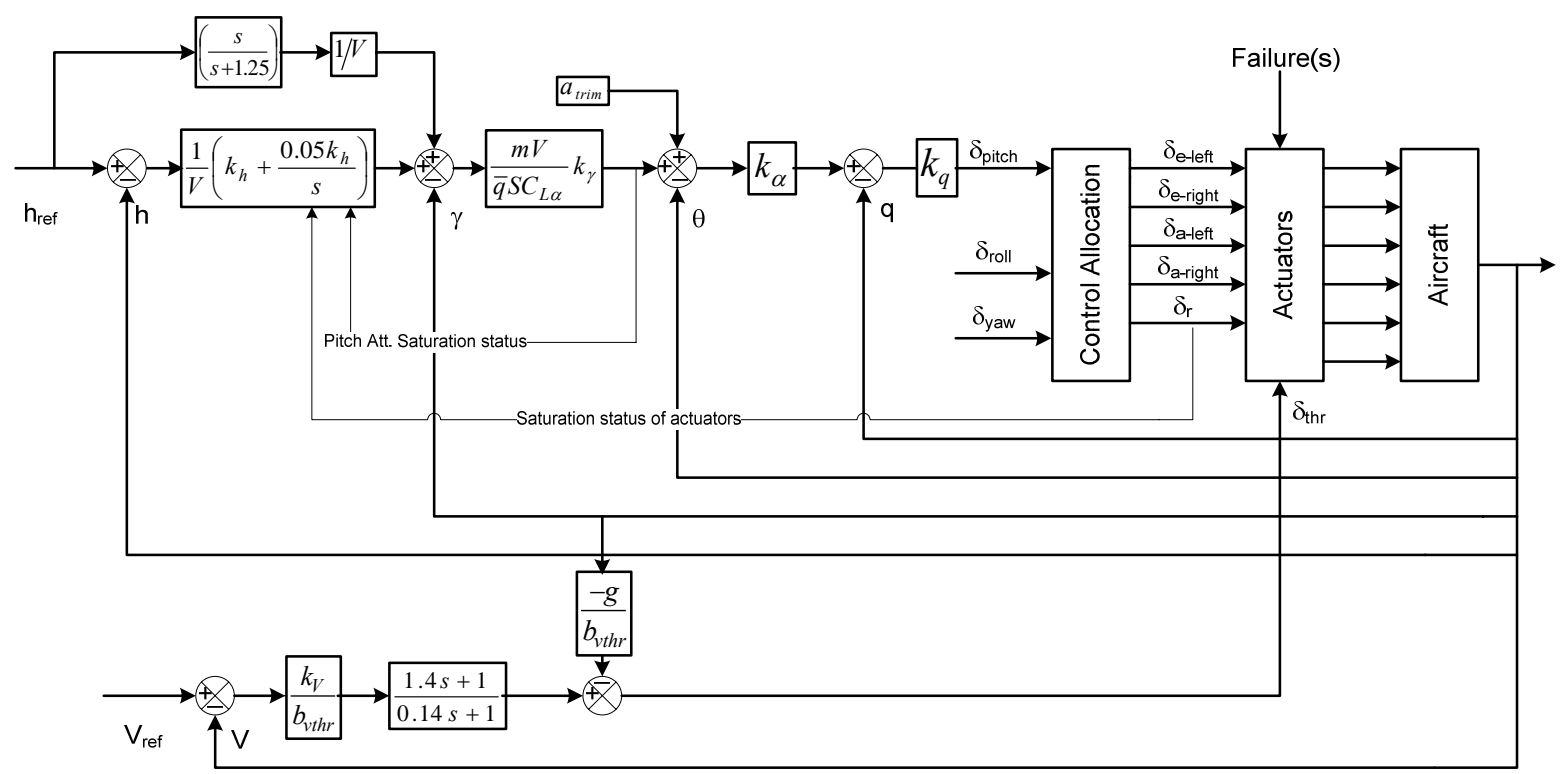

Fig. 2 Longitudinal Axis Feedback Controller (FC) 


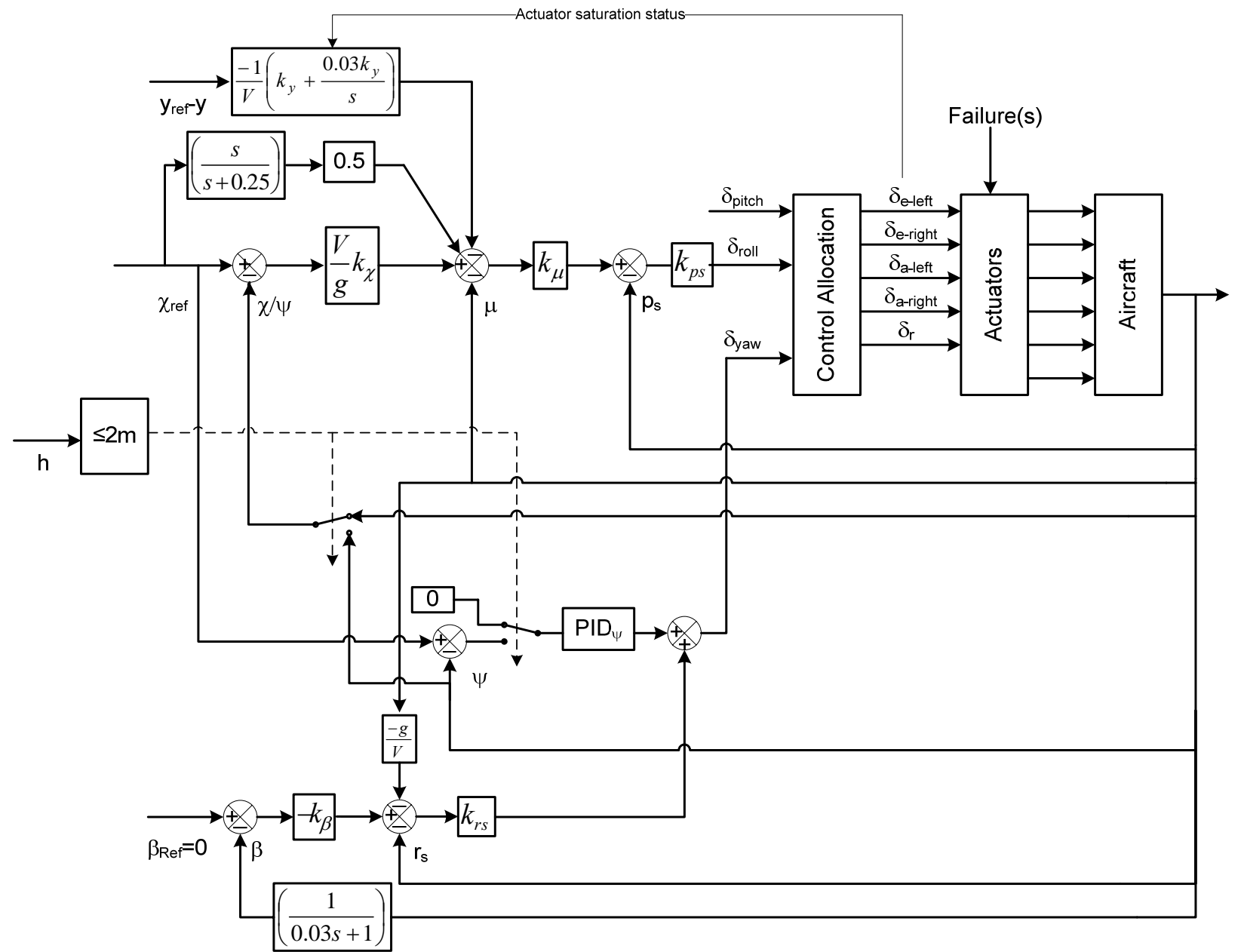

Fig. 3 lateral-Directional Axis Feedback Controller (FC) 


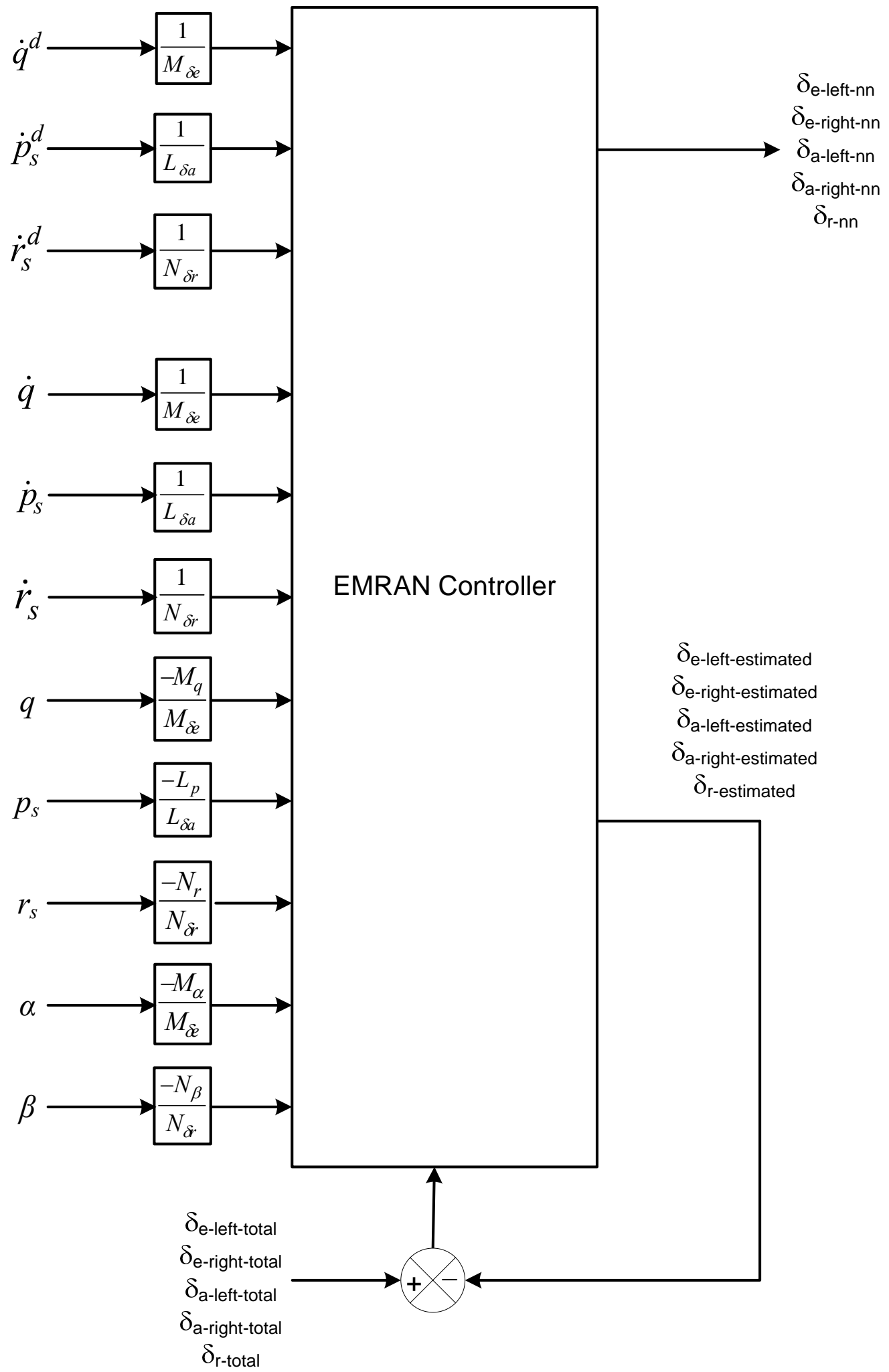

Fig. 4 EMRAN Controller Block Schematic 


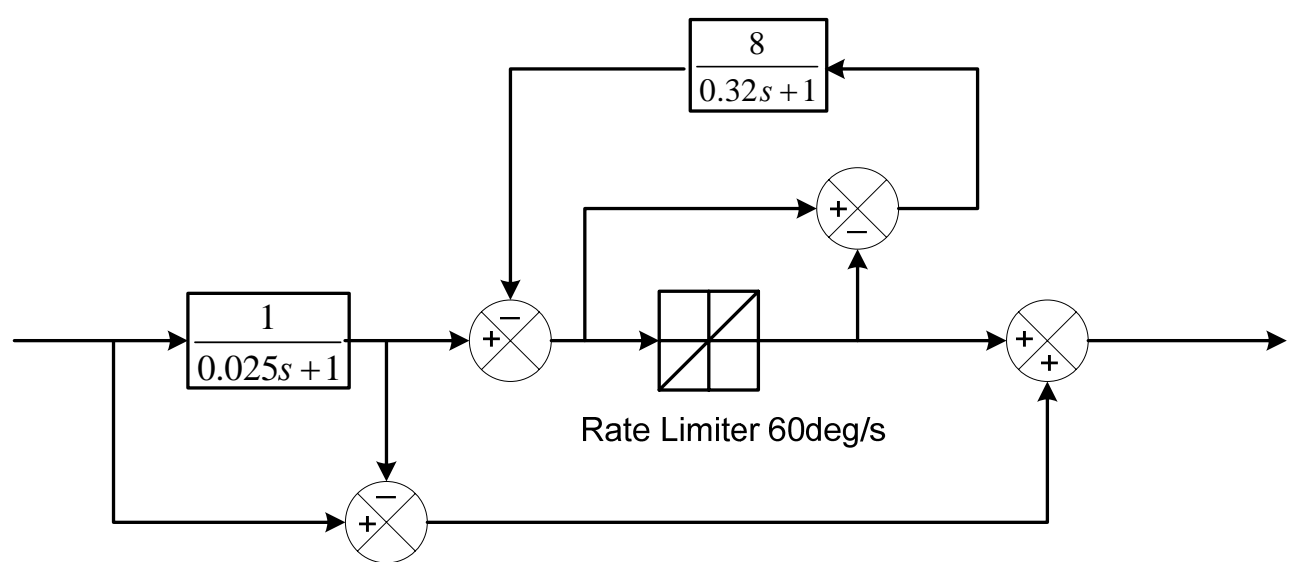

Fig. 5 SAAB Phase Compensator for Alleviation of Actuator Rate Limiting 

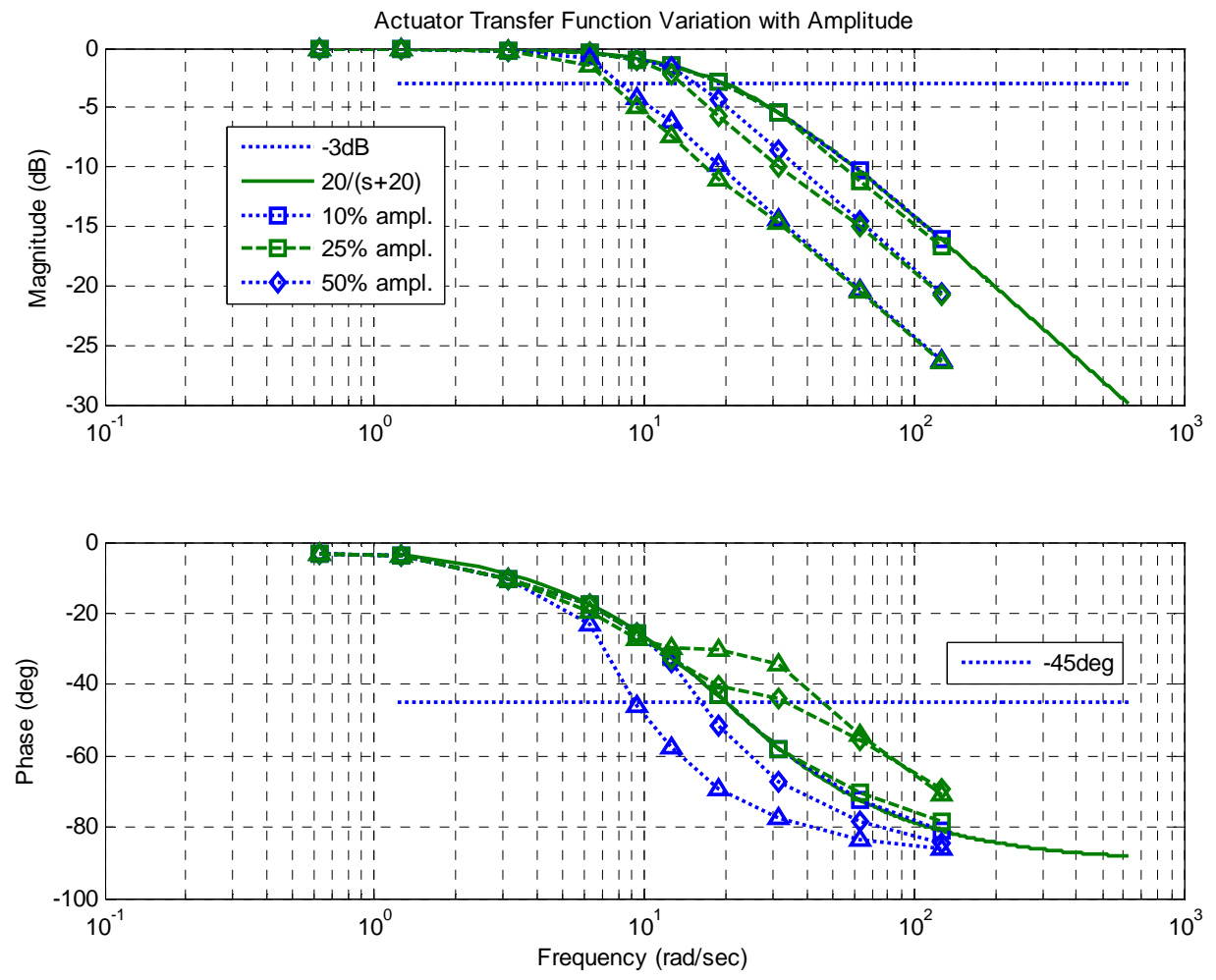

Fig. 6 Amplitude dependent frequency response of actuator.

Dotted lines (without compensator), Dashed lines (with compensator) 


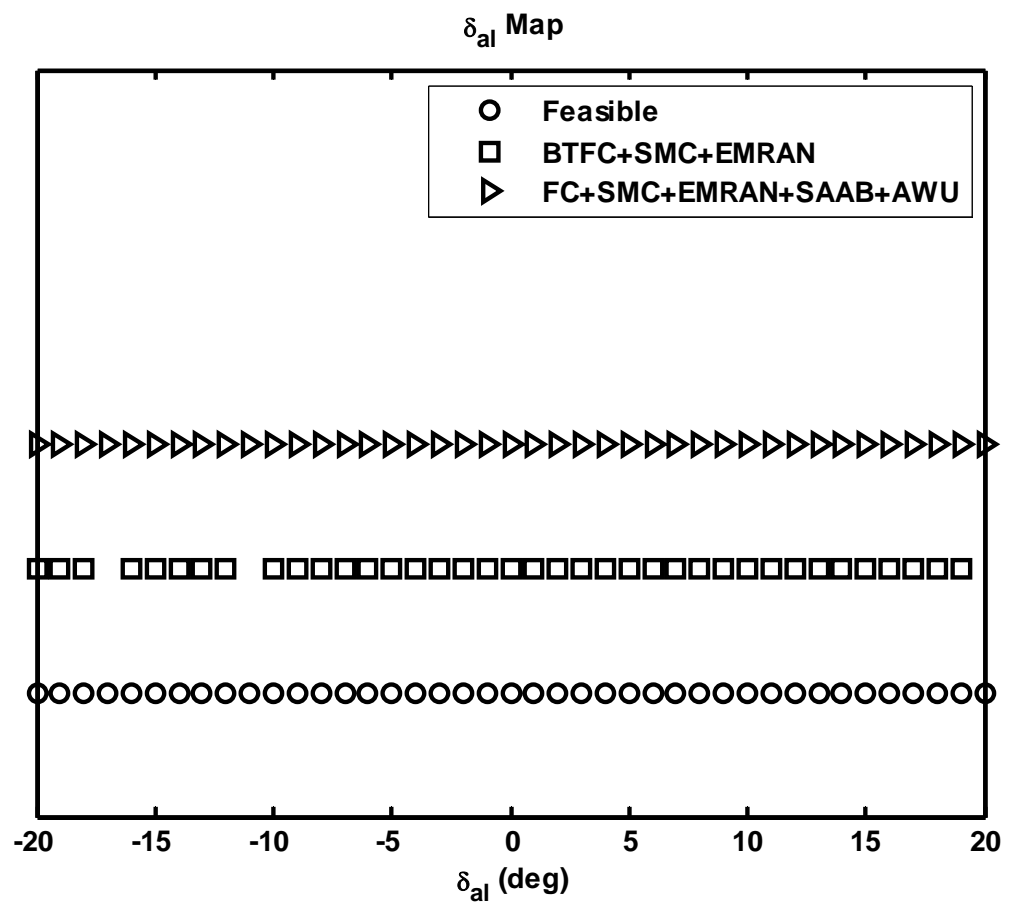

Fig. 7 Left-aileron fault tolerance feasibility map for BTFC+SMC+EMRAN (open squares) and for FC+SMC+EMRAN+SAAB+AWU (open triangles) 


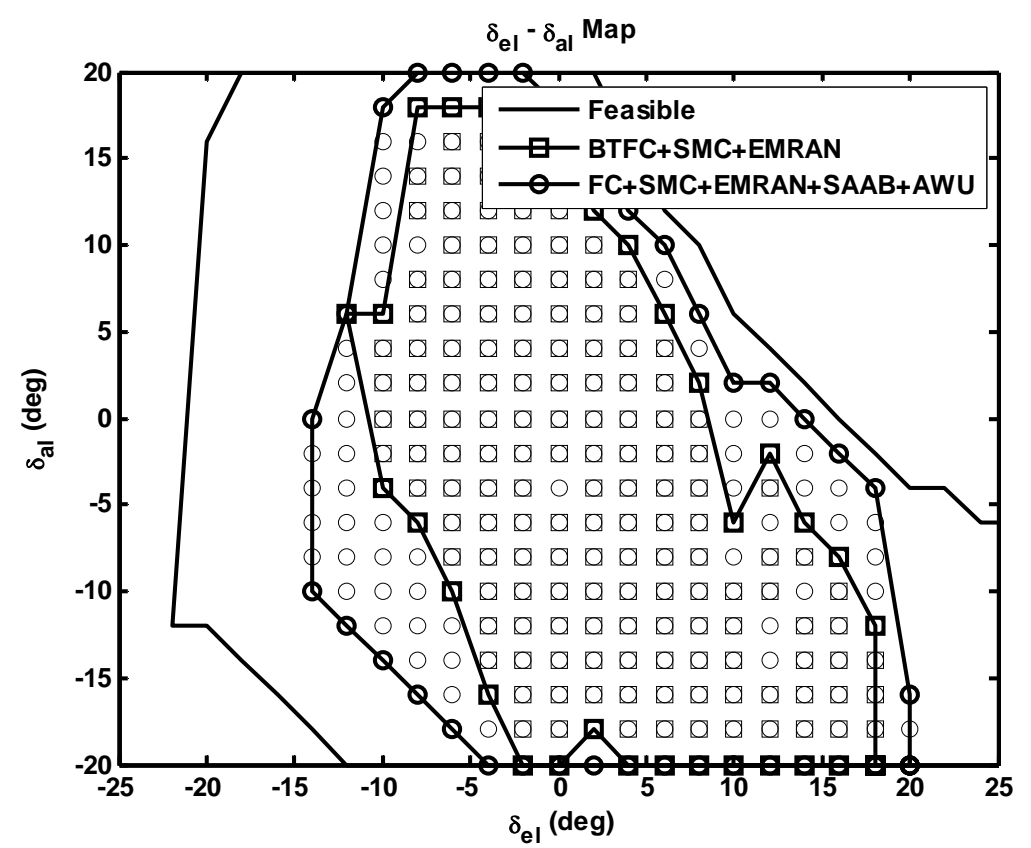

Fig. 8 Left-elevator and left-aileron fault tolerance feasibility map for BTFC+SMC+EMRAN (open squares) and for FC+SMC+EMRAN+SAAB+AWU (open circles) 

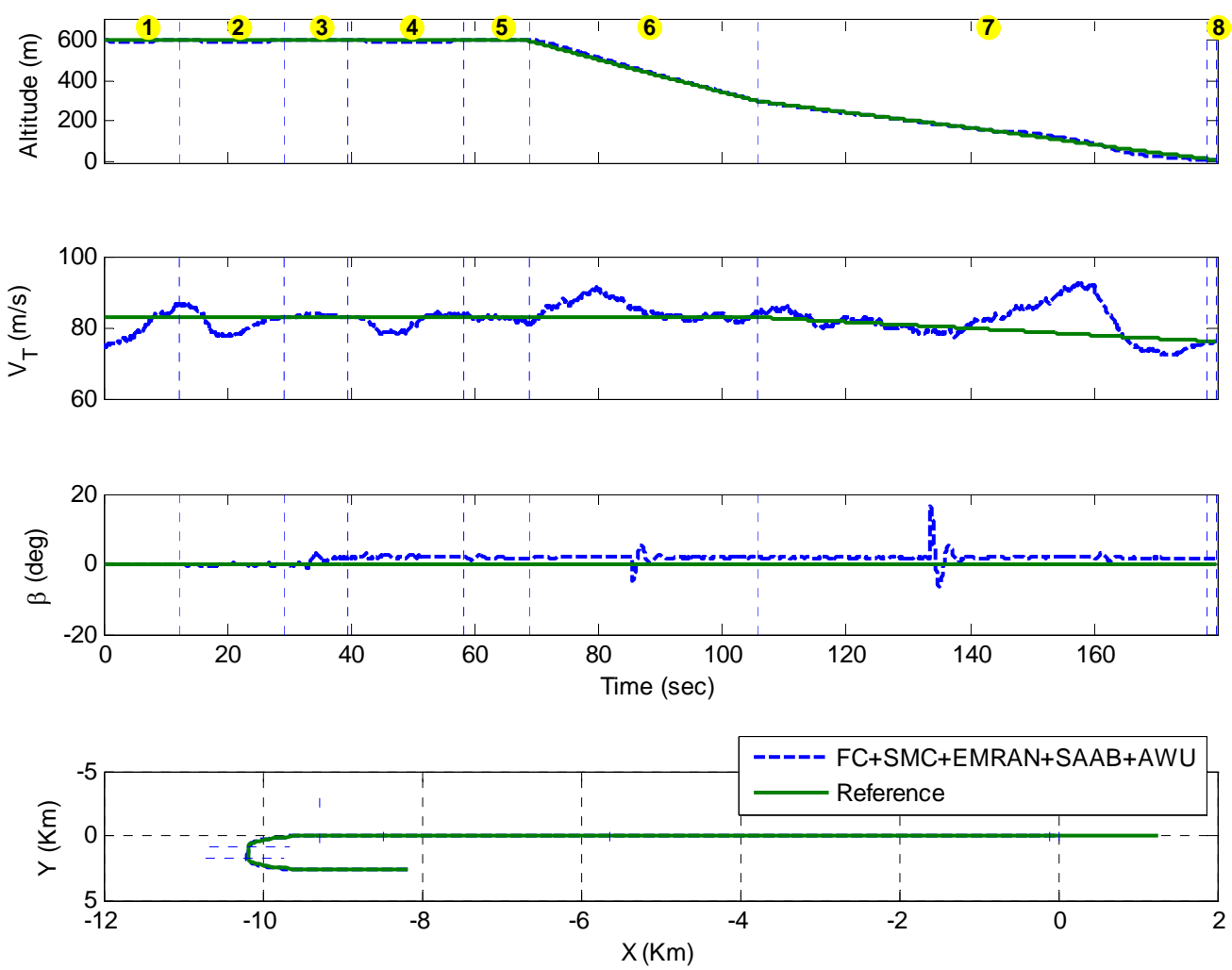

Fig. 9 Aircraft response for a three failures case (left aileron stuck at $2 \mathrm{deg}$, right aileron stuck at $\mathbf{- 2} \mathrm{deg}$, and rudder stuck at $4 \mathrm{deg}$ ) 